\title{
Real-Time Quality Control of Tracked Ultrasound
}

\author{
Emad M. Boctor, Iulian Iordachita, Gabor Fichtinger, and Gregory D. Hager \\ Engineering Research Center, Johns Hopkins University \\ eboctoraieee.org, iordachita jhu.edu, gabor@cs jhu.edu, \\ hager@cs. jhu.edu
}

\begin{abstract}
The overwhelming majority of intra-operative hazard situations in tracked ultrasound (US) systems are attributed to failure of registration between tracking and imaging coordinate frames. We introduce a novel methodology for real-time in-vivo quality control of tracked US systems, in order to capture registration failures during the clinical procedure. In effect, we dynamically recalibrate the tracked US system for rotation, scale factor, and in-plane position offset up to a scale factor. We detect any unexpected change in these parameters through capturing discrepancies in the resulting calibration matrix, thereby assuring quality (accuracy and consistency) of the tracked system. No phantom is used for the recalibration. We perform the task of quality control in the background, transparently to the clinical user while the subject is being scanned. We present the concept, mathematical formulation, and experimental evaluation in-vitro. This new method can play an important role in guaranteeing accurate, consistent, and reliable performance of tracked ultrasound.
\end{abstract}

\section{Background and Significance}

Ultrasound (US) imaging has become a widely accepted guidance modality in medical interventions, because it is real-time, safe, and of low cost. Significant research has been dedicated to quantitative tracked ultrasound, involving tracking the US probe in $3 \mathrm{D}$ space with respect to a stationary frame of reference. While tracked US originates from interventional applications, it recently has become an indispensable tool in external beam radiation therapy (EBRT) guidance [1] that is expected to become the largest user of tracked ultrasound in the next couple of years. Each year in the United States 65,000 patients are treated for prostate cancer alone. Considering an average of 40 treatment fractions that each patient receives, the total number of procedures accumulates to 2.6 million a year. Tracked US guidance is also applicable to the EBRT of breast cancer, adding about 2 million more cases to the potential market.

Typically, tracking is achieved by rigidly attaching 3D localizers to the US probe. The missing link, however, is the spatial transformation between the US image pixels and the $3 \mathrm{D}$ localizers on the probe, which requires calibration. Hence, calibration is ubiquitously present in all systems where ultrasound is used for quantitative image guidance. From our experience, the wide majority of intra-operative hazard situations in tracked US systems are caused by failure of registration between tracking and imaging coordinate frames, thereby manifesting in miscalibration of the tracked US. 
The most typical form of errors is false reading of the tracker that occurs quite often in electromagnetic tracking systems due to invisible field distortions caused by metal objects or electromagnetic noise. Another typical problem related to tracking is deformation or physical damage of the tracking body attached to the probe, causing a latent misreading of pose. What makes these problems exceedingly dangerous is that they occur without apparent warning. Among human operator errors, inadvertent changes of lateral image polarity occur quite frequently and transparently to the clinical user. With regular off-line recalibration some of the aforementioned errors can be caught prior to procedure. The process is called Quality Control (Q/C), a mandatory routine in any clinical department. Typically, $\mathrm{Q} / \mathrm{C}$ is performed annually, monthly, or weekly, which places a heavy financial burden on the department. In addition to increasing patient safety, reduction in $\mathrm{Q} / \mathrm{C}$ costs clearly is an incentive.

In all current calibration methods, a set of objects (often referred to as phantoms) of known geometrical properties are scanned and then various mathematical procedures are applied to discern the unknown transformation that maximizes the similarity between the US images and the phantom [3-6]. The use of special objects and phantoms, however, is cumbersome and foreign to the operating room where interrupting the procedure for the sake of probe calibration is not practical. What is required therefore is a paradigm shift in calibration technology to phantomless selfcalibration that is performed directly on the patient, intra-operatively, in real-time, transparently to the physician.

Generally, full calibration involves six degrees-of-freedom (DOF) of rigid transformation and three dimensional scaling. However, according to a practical observation, the possibility of pure translational error in the calibration matrix is practically low. One atypical scenario would involve the rigidly attached sensor to slide without experiencing any relative rotation with US image reference frame. Thus for the purpose of $\mathrm{Q} / \mathrm{C}$, it is sufficient to recalibrate the system for the remaining degrees-offreedom. This paper presents the concept, mathematical framework, experimental implementation, and in-vitro evaluation of a phantomless real-time method that detects intra-operative failures of the tracked US while recovering the calibration matrix to a $2 \mathrm{D}$ scale factor and then achieve full calibration in an additional step.

\section{Mathematical Formulation}

The key enabler of our self-calibration method is a closed-form mathematical formulation of the problem. Fig. 1 presents the coordinate systems for the mathematical formulation. $A_{1}, A_{2}$ are the transformations of US picture coordinate system $(P)$ with respect to the fixed construction frame $(C)$ at poses 1 and 2, respectively. Note that the actual selection of $\mathrm{C}$ is arbitrary and the only requirement is that it must be rigidly fixed during the calibration process. Using $A_{l}$ and $A_{2}$, we obtain the transformation between poses 1 and 2 , as $A=A_{2} A_{l}^{-1}$. At the same time, the transformation between the two poses can be recovered using

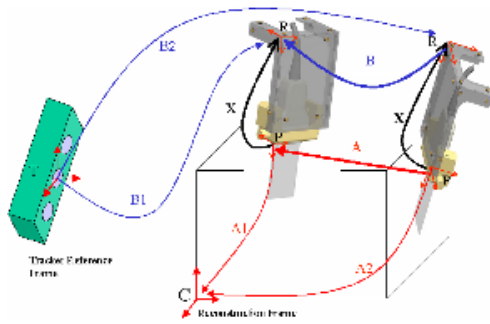

Fig. 1. Coordinate definitions in the closed form $A X=X B$ 
a calibration phantom or recovered directly by matching the $2 \mathrm{D}$ ultrasound images acquired in these poses to a prior $3 \mathrm{D}$ model of the phantom object. $B_{1}$ and $B_{2}$ are readings from the tracker for the sensor frame $(R)$ with respect to tracker reference frame $(T)$, at poses 1 and 2 respectively. The relative pose between the sensor frame $(R)$ at pose 1 and 2 is given by $B=B_{2}^{-1} B_{l}$. This yields the following homogeneous matrix equation:

$$
\begin{aligned}
& A X=X B, \text { which is : } \\
& R_{a} R_{x}=R_{x} R_{b}, \text { and } \\
& R_{a} t_{x}+D\left(u_{a}\right) \bar{\lambda}=R_{x} t_{b}+t_{x}
\end{aligned}
$$

Where $A$ is estimated from images, $B$ is assumed to be known from the external tracking device, and $X$ is the unknown transformation between the US image coordinate system and the sensor frame $(R)$. Expanding this equation yields two separate constraints on translation and rotation, where $R_{a}$ is the rotation of the US image frame between pose 1 and $2, \lambda$ is the unknown scale factor vector that relates the translation vector $u_{a}$ in voxel space to the US image frame translation vector $t_{a}$ (usually expressed in $\mathrm{mm}$ ), such that:

$$
t_{a}=\left(\begin{array}{l}
\lambda_{x} u_{a x} \\
\lambda_{y} u_{a y} \\
\lambda_{z} u_{a z}
\end{array}\right)=\left(\begin{array}{ccc}
u_{a x} & 0 & 0 \\
0 & u_{a y} & 0 \\
0 & 0 & u_{a z}
\end{array}\right) \cdot\left(\begin{array}{l}
\lambda_{x} \\
\lambda_{y} \\
\lambda_{z}
\end{array}\right)=D\left(u_{a}\right) \vec{\lambda} .
$$

It is important to account for the most general case where the scale factor $\lambda$ (which again converts from voxel space to image space) is not known. This scenario typically occurs when $A$ is recovered by registering the US image to some a priori known model (or phantom) given in voxel space. In the linear formulation of the problem, we use the linear operator vec and the Kronecker product $(\otimes)$ [8]. Using the following property of the Kronecker product $\operatorname{vec}(C D E)=\left(C \otimes E^{T}\right) \operatorname{vec}(D)$, we rewrite Eq. 1 as:

$$
\begin{aligned}
& \left(R_{a} \otimes R_{b}\right) \operatorname{vec}\left(R_{x}\right)=\operatorname{vec}\left(R_{x}\right), \text { and } \\
& \left(I_{3} \otimes t_{b}^{t}\right) \operatorname{vec}\left(R_{x}\right)+\left(I_{3}-R_{a}\right) t_{x}-D_{u} \lambda=0
\end{aligned}
$$

This leads to a homogeneou s linear system

$$
\left[\begin{array}{ccc}
I_{9}-R_{a} \otimes R_{b} & 0_{9 * 3} & 0_{9 * 3} \\
I_{3} \otimes t_{b}^{t} & I_{3}-R_{a} & -D_{u}
\end{array}\right]\left(\begin{array}{c}
\operatorname{vec}\left(R_{x}\right) \\
t_{x} \\
\lambda_{3 * 1}
\end{array}\right)=\left(\begin{array}{c}
0_{9 * 1} \\
0_{3^{*} 1}
\end{array}\right)
$$

The solution for this homogeneous linear system could be given by finding the null space, which is a subspace in $R^{15}$. Then the unique solution could be extracted from the null space using the unity constraint to the first 9 coefficients representing the $R_{x}$. However, a better solution is described in [9] where the system is solved in two steps: first extract the rotation, and then solve for the translation and scale. The complete algebraic analysis for this problem (where the scale factor is assumed to be the same in three directions) is given in [7], where it is proved that two independent motions with non-parallel axes is sufficient to recover a unique solution for $A X=X B$. We have extended this solution method to account for inhomogeneous scale in the three coordinate axes. As discussed in [5], two independent motions with $A$ 's and $B$ 's are sufficient to recover $X$. Therefore, our present task reduces to recover $A$ 's as we are scanning real tissue and collecting the corresponding $B$ 's from the tracker, and then to obtain the calibration by solving the homogenous linear system in Eq. 3. The 
challenge lays in the full recovery of the $A$ 's, including all six DOF. The in-plane motion parameters can be recovered with sub-pixel accuracy in real-time, using speckle based tracking algorithms applied to the B-mode images. The out-of-plane motion parameters are difficult to recover from B-mode and we performed this task only with RF or pseudo-RF data, the latter being decompressed B-mode data as described by Prager et al. [13]. Out-of-plane motion, however, is not necessary for our immediate task of $\mathrm{Q} / \mathrm{C}$, thus it is omitted from further discussion in this paper.

\section{Translation Motion:}

This motion scenario is realized by moving the 2D/3D US probe in translational sweep (without rotation) to collect nearly parallel stack of images and/or series of 3D slabs. Also it can be shown in a panoramic scan where the images can be stitched together without introducing a relative rotation. Given this kind of motion, $R b=R a=I_{3}$, leading to $R x * t_{b i}=t_{a i}$, where $i$ denotes the motion. Using the property of the Kronecker product used in Eq. 1:

$$
\begin{aligned}
& \left(I_{3} \otimes t_{b i}^{T}\right) *_{9 * 1} \operatorname{vec}(R x)=t_{a i}, \text { we have } \\
& t_{a i}=\left[\begin{array}{c}
\lambda_{x} * u_{a i}(x) \\
\lambda_{y} * u_{a i}(y) \\
\lambda_{z} * u_{a i}(z)
\end{array}\right]=\left[\begin{array}{ccc}
u_{a i}(x) & 0 & 0 \\
0 & u_{a i}(y) & 0 \\
0 & 0 & u_{a i}(z)
\end{array}\right]\left[\begin{array}{l}
\lambda_{x} \\
\lambda_{y} \\
\lambda_{z}
\end{array}\right]=D\left(u_{a i}\right) \vec{\lambda}
\end{aligned}
$$

Three independent translations are sufficient to obtain a full-rank system. Solving for $R x$ and the three scale factors:

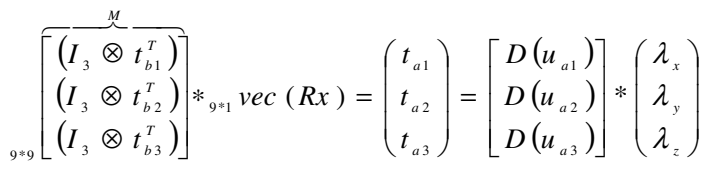

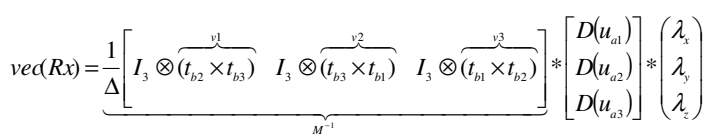

Using Khatri-Rao product [8] which defined as:

${ }_{q \times u} F_{\circ^{\prime \times u}} G=\left[\begin{array}{llll}F_{1} \otimes G_{1} & F_{2} \otimes G_{2} & \cdots & F_{u} \otimes G_{u}\end{array}\right]$, and the following fact $\operatorname{vec}(\mathrm{AVD})=\left(\mathrm{D}^{\mathrm{T}} \circ \mathrm{A}\right) \operatorname{vecd}(\mathrm{V})$, vecd is a diagonal vec operator

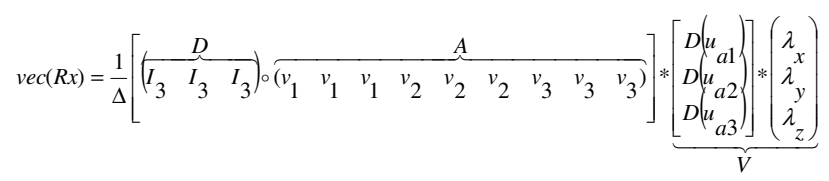

$\operatorname{vec}(R x)=\frac{1}{\Delta} \operatorname{vec}\left(A^{*} V^{*} D^{T}\right)$, fromlinearity of vecoperator

$$
R x=\frac{1}{\Delta} A^{*} V^{*} D^{T}=\frac{1}{\Delta}\left[\begin{array}{c}
\lambda_{x}\left(u_{a 1}(x) v_{1}+u_{a 2}(x) v_{2}+u_{a 3}(x) v_{3}\right) \\
\lambda_{y}^{x}\left(u_{a 1}(y) v_{1}+u_{a 2}(y) v_{2}+u_{a 3}(y) v_{3}\right) \\
\lambda_{z}\left(u_{a 1}(z) v_{1}+u_{a 2}(z) v_{2}+u_{a 3}(z) v_{3}\right)
\end{array}\right]^{T}
$$


We have obtained a closed-form solution that recovers an anisotropically scaled rotation matrix from three independent translations. We can recover the three scale factors by applying the unit constraint on each column vector, and recover the underlying rotation by then rescaling the columns to be unit vectors. This basically proves that three independent motions are sufficient to recover the rotation and the three scale factors. However, the third motion is not even necessity. It can be shown [7] that given two independent motions $t_{b 1}, t_{b 2}$, the third constraint comes from the cross-product between $t_{b 1}, t_{b 2}$.

In order to map this analysis to our application, several requirements must be considered. The ultrasound machine generates real-time 2D US pixel-map, meaning that we have only two unknown scale factors in $x$ and $y$, denoted as $\lambda_{\mathrm{x}}$ and $\lambda_{\mathrm{y}}$. With sweeping probe motion we obtain multiple poses that suggests folding the closed form representation into a least squares problem. Starting from the following equation:

$$
\begin{aligned}
& R x * t_{b i}=t_{a i}, \text { we have } \\
& t_{a i}=\left[\begin{array}{c}
\lambda_{x} * u_{a i}(x) \\
\lambda_{y} * u_{a i}(y) \\
0
\end{array}\right]=\left[\begin{array}{ccc}
\lambda_{x} & 0 & 0 \\
0 & \lambda_{y} & 0 \\
0 & 0 & \lambda_{z}
\end{array}\right]\left[\begin{array}{c}
u_{a i}(x) \\
u_{a i}(y) \\
0
\end{array}\right]=D(\vec{\lambda}) u_{a i} \\
& {\left[\begin{array}{llll}
t_{b 1} & t_{b 2} & \cdots & t_{b n}
\end{array}\right]=R x^{T} D(\vec{\lambda})\left[\begin{array}{llll}
u_{a 1} & u_{a 2} & \cdots & u_{a n}
\end{array}\right]}
\end{aligned}
$$

The solution of this equation can be achieved in many ways. One can solve nonlinearly for the three rotations and the two scale factors $\left(\lambda_{\mathrm{x}}, \lambda_{\mathrm{y}}\right)$. Alternatively, one can solve linearly for the nine parameters of the scaled rotation and perform $Q R$ factorization with positive scale factor constraints. Or simply, apply the norm constraint on Eq. 4 as follows:

$$
\left\|R x * t_{b i}\right\|^{2}=\left\|t_{b i}\right\|^{2}=\left\|D(\vec{\lambda}) u_{a i}\right\|^{2}=u_{a i}^{T} D(\vec{\lambda})^{2} u_{a i}
$$

This allows solving for both $\left(\lambda_{\mathrm{x}}, \lambda_{\mathrm{y}}\right)$. After recalculating the scaled $u_{a i}$, we obtain two sets of points governed by an $\mathrm{SO}(3)$ rotation and recovered with Horn's method[11].

\section{Planar Motion:}

Planar motion is more general as it also allows, in addition to translation, in-plane rotation. This type of sweeping probe motion is commonly applied clinically. With this general motion, one can recover both rotation and anisotropic scale factors as shown before, but it will not yield a full recovery of the position offset $t_{x}$. By applying Eq. 3 we obtain:

$$
\begin{gathered}
{\left[\begin{array}{cc}
I_{9}-R_{a i} \otimes R_{b i} & 0_{9 * 3} \\
I_{3} \otimes t_{b i}^{t} & -D_{u i} \\
\vdots &
\end{array}\right]\left(\begin{array}{c}
\operatorname{vec}\left(R_{x}\right) \\
\lambda_{3 * 1}
\end{array}\right)=\left(\begin{array}{c}
0_{9+1} \\
0_{3 * 1+1} \\
\vdots
\end{array}\right) \text { and }} \\
{\left[\begin{array}{c}
\left(I_{3}-R_{a i}\right) \\
\vdots
\end{array}\right] t_{x}=\left[\begin{array}{c}
D_{u i} \vec{\lambda}_{3 * 1}-\left(I_{3} \otimes t_{b i}^{t}\right) \operatorname{vec}\left(R_{x}\right) \\
\vdots
\end{array}\right]}
\end{gathered}
$$

Note that when $R_{b}=R_{a}=I_{3}$, Eq. 5 becomes similar to the pure translation case in Eq. 4 . Also note that Eq. 6 is always under constraint as $\left(I_{3}-R_{a}\right)$ has rank 2, regardless to the number of in-plane rotations, meaning there is no single solution for $t_{x}$ and the general solution will have exactly one (the number of unknowns minus the rank) arbitrary scale factor $\zeta$. So the solution can take the form: 


$$
t_{x}(\zeta)=t_{\mathrm{o}}+\zeta * t_{n}
$$

Where $\zeta$ is the unknown scale factor. $t$ 。 is a unique solution in the plane of motion (2dimensional), since $\left(I_{3}-R_{a}\right)$ has rank 2. $t_{n}$ is the normal to the plane of motion. In our case, if the plane of motion is the US image plane (the $\mathrm{x}-\mathrm{y}$ plane), $t_{n}$ may equal ( $\begin{array}{lll}0 & 0\end{array}$ $1)^{\mathrm{T}}$, which is a unit vector in the z-direction and thus perpendicular to the plane of motion. In our US calibration and $\mathrm{Q} / \mathrm{C}$ system, we can recover this unknown $\zeta$, if we have a collection of a previously scanned cross-wire tar get from different insonification angles, the idea being is that the cloud of reconstructed cross-wire targets will be the smallest (i.e. standard deviation minimal) for the correct value for $\zeta$.

\section{Experimental System}

In our experimental prototype (Fig. 2), we used a SONOLINE Antares US scanner (Siemens Medical Solutions USA, Inc. Ultrasound Division, Issaquah, WA), with a Siemens VF 10-5 linear array probe. The patient was replaced with a tissue mimicking agar phantom. The US probe was held against the phantom in a rigid acrylic holder mounted on an adjustable steady arm. The arm had dual purpose: to adjust the spatial position of the tracked US probe over the calibration phantom, and to ensure temporal synchronization between the tracker and the US scanner. Multiple optical markers were attached to the probe holder, which then were tracked by an OPTOTRAK device (Northern Digital Inc.).

The tissue mimicking phantom provides realistic images of fully developed speckles. Its construction is based on a recipe by Fenster et al. [10]. Three percent by weight of agar gel (A-7002 Agar, Sigma-Aldrich, St. Louis, MO) was added to distilled water, with three percent by weight $50 \mu \mathrm{m}$ cellulose particles (S-5504 Sigmacell, Sigma-Aldrich), and with seven percent by volume glycerol (W25250, Sigma-Aldrich). The mixture was heated to $92 \mathrm{C}^{\circ}$, stirred constantly, gradually cooled to $60 \mathrm{C}^{\circ}$, and then poured into a container mold. We also introduced specular features and structures to mimic bone appearance and to allow for testing algorithmic performance under different echogenicity conditions.

\section{Algorithm Workflow}

The workflow in the real-time $\mathrm{Q} / \mathrm{C}$ procedure is described in Fig 3. The Acquisition Module

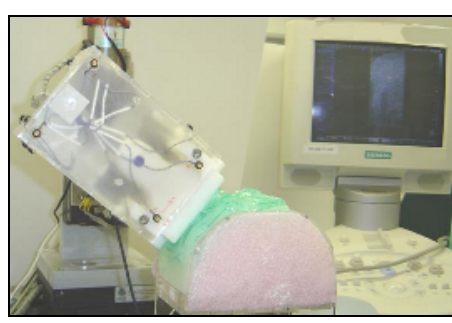

Fig. 2. In-vitro experimental setup

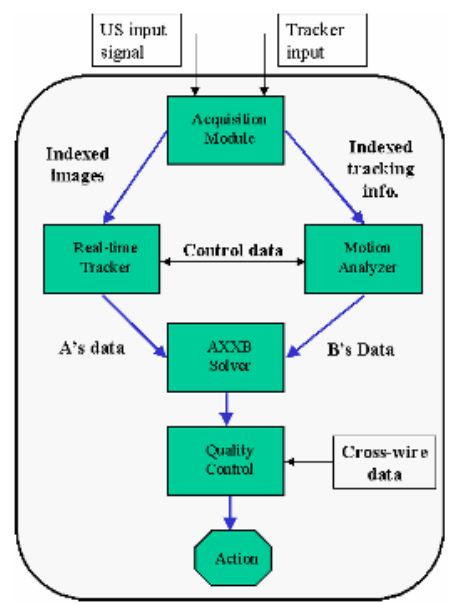

Fig. 3. The Q/C workflow receives US video signal and tracker reading, from which it prepares synchronized indexed sequences of images and tracking information. The Motion Analyzer sorts out 
the types of motions in these sequences and sends control signal for the Real-time Tracker, which recovers the $A$ matrices. Finally, the $A X=X B$ solver receives corresponding $A$ 's and $B$ 's data, recovers the $X$ calibration matrix. The Quality Control unit analyzes the new calibration and compares it with previous runs. In case of suspected discrepancy, an appropriate Action is initiated to deal with the hazard condition. The action could range from generating a warning message to demanding to halt the procedure and fully recalibrate the system. It must be noted that a full recalibration is necessary only if a scale factor is found to be at fault, because the partial calibration recovers all other parameters during the $\mathrm{Q} / \mathrm{C}$ process. The remaining scale factor can be easily recovered from a pre-scanned geometrical object, such as a cross-wire, as described earlier in relation to Eq. 7.

\section{Real-time Tracker}

As mentioned above, the role of Real-time Tracker is to recover the A matrices, the motion of the US image in construction frame, as it was described in Fig. 1. What is necessary is to compute the relative motion in pairs of ultrasound images for which the absolute (tracked) motion is known. We accomplish this using direct image registration methods similar to those described in [12]. Specifically, we introduce an intermediate "warped" image representation $W$ defined as

$$
W(u, t ; p, \alpha)=I(\operatorname{Rot}(\alpha) * u+p)
$$

where $u=(x, y)^{T}$ is an image location, $P$ is a translation offset, and $\alpha$ is an interframe rotation. Let $W(t ; p, \alpha)$ denote the column vector constructed by stacking the value of $W$ for all possible image locations $u$. We then compute an estimate of the offset $\left(P_{t}\right.$, $\alpha_{t}$ ) between images at time $t$ and $t+d$ by iterating the following equation:

$$
\left(p_{t}^{k}, \alpha_{t}^{k}\right)=\left(p_{t}^{k-1}, \alpha_{t}^{k-1}\right)+J^{\mp}\left(W\left(d+t ; p_{t}^{k-1}, \alpha_{t}^{k-1}\right)-W(t ; 0,0)\right)
$$

where $J^{\mu}$ denotes the pseudo-inverse of the Jacobian matrix of $W$ with respect to $P$ and $\alpha$. The values of $\left(P_{t}, \alpha_{t}\right)$ is taken from the previous frame.

\section{Experiments and Results}

We used the experimental system to collect 5 datasets, altogether containing 20 motions in B-mode, of rectangular view and $8 \mathrm{~cm}$ depth. One of the datasets contained 4 motions and was obtained under a faulty condition. We purposely flipped lateral polarity of the Bmode image to simulate a common operator error. Table 1 shows the rotation and scale reported by the $\mathrm{Q} / \mathrm{C}$ system. In testing the image tracker, we used $d=10$ step size, for
Table 1. Q/C system report on rotation and scale

\begin{tabular}{l|c|l|l|} 
& $\begin{array}{c}\text { Magnitude of } \\
\text { rotation } \\
\text { (Rodrigues } \\
\text { form) }\end{array}$ & \multicolumn{2}{|c|}{$\begin{array}{c}\text { Scale in x and y } \\
\text { (mm/pixel) }\end{array}$} \\
\hline Dataset-1 & 3.163 & 0.22 & 0.26 \\
\hline Dataset-2 & 3.072 & 0.23 & 0.26 \\
\hline Dataset-3 & 2.992 & 0.23 & 0.27 \\
\hline Dataset-4 & 3.008 & 0.23 & 0.29 \\
\hline Dataset-5 & 0.086 & 0.24 & 0.27 \\
\hline
\end{tabular}


which images were matched with an RMS gray-scale error of less than $2 \%$ of signal value, suggesting a registration error of less than $1 / 10$ pixel [12]. The heart of the $\mathrm{Q} / \mathrm{C}$ system, as seen in Fig. 3, are the real-time image tracker and $A X=X B$ solver. The image tracker is sensitive to the step $d$ between registered images, while the $A X=X B$ solver is sensitive to the number and type of motions used to recover the calibration parameters. The upper graph in Fig. 4 illustrates the relation between the recovered scale and number of images and the step size $d$ taken in the image registration algorithm, for "bad" (appears in the upper graph for the first 30 images) and "good" (lower graph). Note that number of images can be represented by the scanning time, where 1 second corresponds to 33 frames, sweeping over a certain distance covered with the given scanning velocity. The $\mathrm{x}$-axis represents the number of frames used in the $A X=X B$ solver and the $y$-axis is the scale ratio in $\mathrm{mm} /$ pixel. The upper figure is for $\lambda_{\mathrm{x}}$, the lower is for $\lambda_{\mathrm{y}}$. Figure 4 also shows convergence for the scale ratio under different image registration steps (small $d: 1,3$, and 5; large $d: 10,20,30$, and 40). Note that as we increase the step size, we also introduce a delay equal to the step size before we start estimating a given parameter. (It is because we must wait for the $d^{\text {th }}$ image to come.)

We have found that steps bigger than 40 are not reliable to track and predict the motions of the speckle-based images. At the same time, they are considered bad kernels, suggesting that we have to wait for about $2 \mathrm{Sec}$. to detect a problem. Yet smaller tracking steps need fewer readings to start convergence, due to the small motions they deliver to the $A X=X B$ solver. Note that the convergence for $d=1,2$, and 3 appears after 20 small motions for $\lambda_{\mathrm{y}}$ and appears after 30 small motions for $\lambda_{\mathrm{x}}$, this is mainly due to the type of motions. The intuition behind this is, we can't estimate a scaling parameter in a direction normal to the direction of motion. Similarly favorable results were obtained for the rotation component, not discussed here due limitation of space. We can conclude that given the right motion, a kernel of 10 steps $(d=10)$ converges in 10-20 steps, meaning 0.3-0.6 sec with a total travel of $\sim 1.5 \mathrm{~mm}$ (scanning speed @ 3mm/sec).
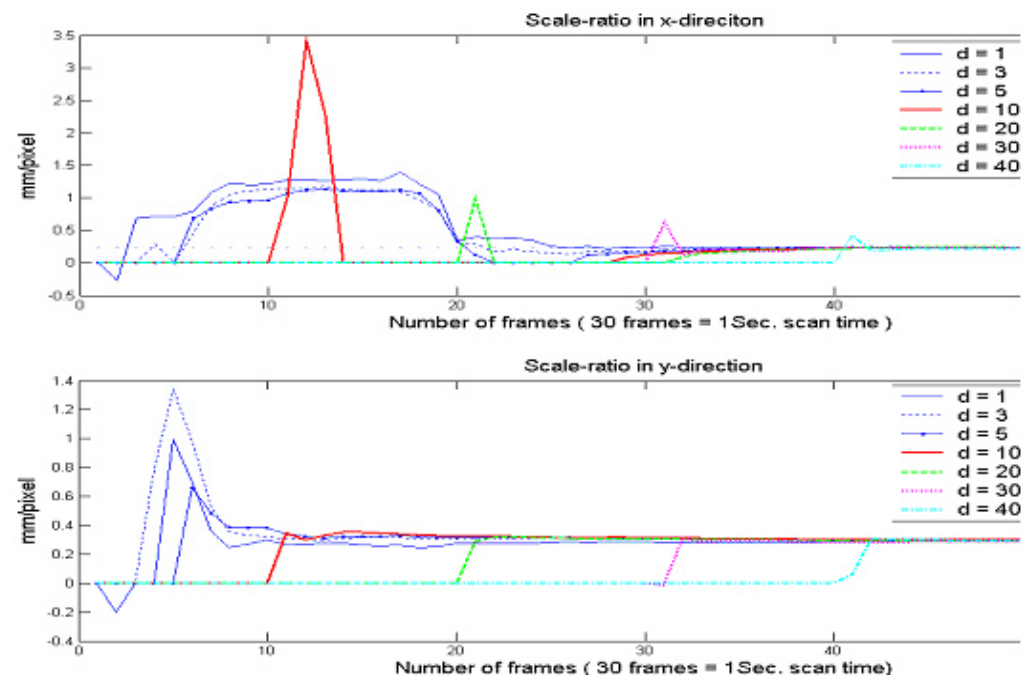

Fig. 4. Recovered scale versus the number of images and step size 


\section{Conclusion and Future Work}

Our Q/C system, that reports the calibration matrix robustly and consistently, recovered the correct calibration parameters under normal working conditions by monitoring the constancy of calibration matrix and did not produce false alarm. It also managed to distinguish the faulty condition through catching the outlier calibration matrix. It is a work in current progress to extend the $\mathrm{Q} / \mathrm{C}$ framework to estimate the out-of-plane motion of the US probe, thereby providing full calibration in-vivo, realtime, as the patient is being scanned, which will obviate phantom-based calibration. The real-time performance of the $\mathrm{Q} / \mathrm{C}$ system allows for averaging hundreds of independent calibrations from a single sweep, which in turn promises to retire crosswire based reconstruction accuracy evaluation and at last make US calibration free from any phantom, whatsoever. The key enabler to this is tuning the Real-time Tracker module to coping with out-of-plane motion components in the $A$ matrix, for which speckle decorrelation techniques are under investigation. We will also compare the accuracy of our current partial recalibration against published off-line calibrations. Our present ability to reproduce calibration parameters to a $2 \mathrm{D}$ scale factor is most promising in this regard. Last but not least, the sensitivity and specificity of the $\mathrm{Q} / \mathrm{C}$ system will be examined. A clinical-grade $\mathrm{Q} / \mathrm{C}$ system must catch all faulty conditions (high sensitivity), yet it must not halt the clinical intervention with producing false alarm (high specificity). Altogether, the combined Q/C and calibration system appears to have high practical utility for clinical departments that use and maintain tracked ultrasound systems. Finally, the authors acknowledge the financial support from the NSF \#EEC 9731478 and loan equipment from Siemens Corporate Research.

\section{References}

1. Langen KM, Pouliot J, Anezinos C, et al. Evaluation of ultrasound-based prostate localization for image-guided radiotherapy, Int J Rad. Oncol Biol Phys. 57(3):635-44.

2. Prager RW, Rohling RN, Gee AH, Berman L. Rapid Calibration for 3-D Freehand Ultrasound, US in Med. Biol., 24(6):855-869, 1998.

3. Pagoulatos N, Haynor DR, Kim Y. A Fast Calibration Method for 3-D Tracking of Ultrasound Images Using a Spatial Localizer, US in Med. Biol., 27(9):1219-1229

4. Rousseau F, Hellier P, Barillot C. A fully automatic calibration procedure for freehand 3D ultrasound, In IEEE Int. Symp. on Biomedical Imaging, 2002.

5. Boctor EM, Viswanathan A, Choti MA, Taylor RH, Fichtinger G, Hager G. A Novel Closed Form Solution for Ultrasound Calibration, In IEEE ISBI 2004.

6. Muratore DM, Galloway RL. Beam calibration without a phantom for creating a 3-D freehand ultrasound system, Ultrasound Med Biol. 2001 Nov;27(11):1557-66.

7. Andreff N, Horaud R, Espiau B. Robot Hand-Eye Calibration Using Structure from Motion, International J. of Robotics Research, 20(3), pp 228-248, 2001.

8. Brewer JW. Kronecker Products and Matrix Calculus in System Theory, IEEE Trans. Circuits and systems, 25(9) Sep.1978.

9. Tsai RY, Lenz RK. A New Technique for Fully Autonomous and Efficient 3D Robotics Hand/Eye Calibration, IEEE Trans. On Robotics and Automation, 5(3):345-358 
10. Rickey DW, Picot PA, Christopher DC, Fenster A. A Wall-less Vessel Phantom for Doppler Ultrasound Studies, US in Med. Biol., 21, 1163-1175, 1995.

11. Horn BKP. Closed-form solution of absolute orientation using unit quaternions, J Opt Soc Am A 1987;4:629-642.

12. Hager G, Belhumeur P. Efficient region tracking with parametric models of geometry and illumination. IEEE PAMI Trans., 20(10):1125-1139, 1998.

13. Prager RW, Gee AH, Treece GM, Berman LH. Decompression and speckle detection for ultrasound images using the homodyned k-distribution. Pattern Reco. Letters 24:705-13. 